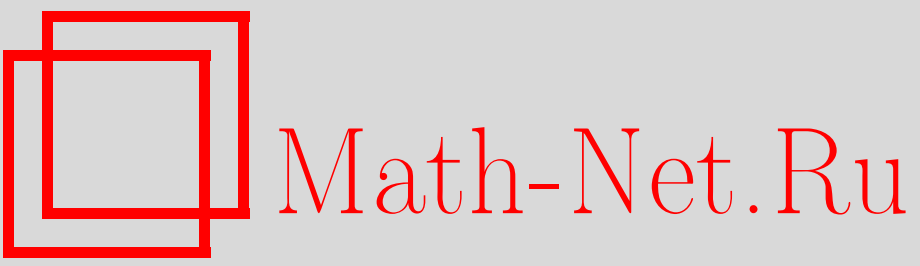

М. В. Булатов, Л. С. Соловарова, Об одном классе квазилинейных дифференциально-алгебраических уравнений второго порядка, Итоги науки и техн. Сер. Соврем. мат. и ее прил. Темат. обз., 2019, том 173, 17-25

DOI: https://doi.org/10.36535/0233-6723-2019-173-17-25

Использование Общероссийского математического портала Math-Net.Ru подразумевает, что вы прочитали и согласны с пользовательским соглашением

http: //www. mathnet.ru/rus/agreement

Параметры загрузки:

IP : 54.162 .85 .209

26 апреля 2023 г., 15:43:16 


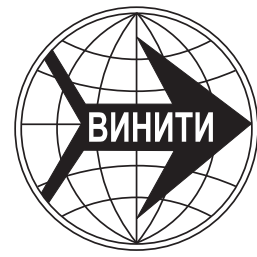

ИТОГИ НАУКИ И ТЕХНИКИ.

Современная математика и ее приложения.

Тематические обзоры.

Том 173 (2019). С. $17-25$

DOI: $10.36535 / 0233-6723-2019-173-17-25$

УДК 517.91

\title{
ОБ ОДНОМ КЛАССЕ КВАЗИЛИНЕЙНЫХ \\ ДИФФЕРЕНЦИАЛЬНО-АЛГЕБРАИЧЕСКИХ УРАВНЕНИЙ ВТОРОГО ПОРЯДКА
}

\author{
(c) 2019 г. $\quad$ М. В. БУЛАТОВ, Л. С. СОЛОВАРОВА
}

\begin{abstract}
АннотАция. Рассмотрены системы квазилинейных обыкновенных дифференциальных уравнений второго порядка с тождественно вырожденной матрицей перед главной частью и с корректно заданными начальными условиями. Отмечено принципиальное отличие таких задач от систем обыкновенных дифференциальных уравнений, разрешенных относительно второй производной. В терминах матричных полиномов сформулированы условия существования и единственности решения данных задач в некоторой окрестности начальной точки.
\end{abstract}

Ключевые слова: дифференциально-алгебраическое уравнение второго порядка, начальная задача, матричный полином.

\section{ON A CERTAIN CLASS OF QUASILINEAR SECOND-ORDER DIFFERENTIAL-ALGEBRAIC EQUATIONS}

\author{
(c) 2019 M. V. BULATOV, L. S. SOLOVAROVA
}

\begin{abstract}
We consider systems of second-order, quasilinear, ordinary differential equations with an identically degenerate matrix coefficient of the principal term and with well-posed initial conditions. Fundamental differences between such problems and systems of ordinary differential equations solved with respect to the second derivative are indicated. In terms of matrix polynomials, we formulate conditions of the existence and uniqueness of solutions of such problems in a neighborhood of the starting point.
\end{abstract}

Keywords and phrases: second-order differential-algebraic equation, initial problem, matrix polynomial.

AMS Subject Classification: 34A09, 65L80

1. Введение. Ряд важных прикладных задач можно описать взаимосвязанными обыкновенными дифференциальными уравнениями (ОДУ) различного порядка и алгебраическими (трансциндентными) уравнениями (см. [6,8]). Такие постановки задач, как правило, записывают в виде системы ОДУ с тождественно вырожденной матрицей перед старшей производной и называют дифференциально-алгебраическими уравнениями (ДАУ). Если порядок хотя бы одного уравнения данной системы имеет порядок 2 и выше, то их называют ДАУ высокого порядка.

$\mathrm{K}$ настоящему времени бурно развивается качественная теория и теория численного решения линейных и квазилинейных ДАУ первого порядка с корректно заданными (согласованными с правой частью) начальными условиями. Для таких постановок задач, особенно для линейных ДАУ с постоянными матричными коэффициентами, фундаментальную роль играет теория матричных

Работа выполнена при поддержке Российского фонда фундаментальных исследований (проекты № 18-51-54001, № 18-01-00643, № 18-29-10019). 
пучков, которая детально изложена в монографии [5]. Однако для ДАУ порядка 2 и выше эта теория применима только для весьма узкого класса задач (об этом будет сказано ниже). Поэтому, как правило, ДАУ высокого порядка записывают путем введения новой вектор-функции в виде ДАУ первого порядка. Такой подход имеет ряд недостатков: увеличивает размерность задачи и «ухудшает» ее свойства. Именно эти факты иициировали исследования, проведенные ниже. Данная статья является продолжением исследований, начатых в [3].

2. Вспомогательные сведения и постановка задачи. Рассмотрим задачу

$$
\begin{gathered}
A(t) x^{\prime \prime}(t)+B(t) x^{\prime}(t)+F(x(t), t)=0, \quad t \in[0,1], \\
x(0)=a,\left.\quad x^{\prime}(t)\right|_{t=0}=b .
\end{gathered}
$$

Здесь и всюду в дальнейшем изложении $A(t), B(t)-(n \times n)$-матрицы, $F(\cdot)-n$-мерная векторфункция.

В данной статье рассмотрены задачи (1), (2) с условием

$$
\operatorname{det} A(t) \equiv 0 \text {. }
$$

Такие задачи будем называть квазилинейными ДАУ второго порядка. Предполагается, что начальные данные (2) заданы корректно, т.е. задача (1)-(2) имеет решение. Под решением мы будем понимать любую вектор-функцию с достаточно гладкими элементами, которая обращает (1) в тождество в некоторой окрестности начальной точки и удовлетворяет условию (2).

Перед изложением особенностей задачи (1)-(2) с условием (3) приведем известные вспомогательные определения и факты.

Определение 1 (см. [5]). Выражение $\lambda A(t)+B(t)$, где $\lambda$-скалярный параметр, $A(t), B(t)-$ $(m \times n)$-матрицы, называется матричным пучком. Пучок называется регулярным, если $m=n$ и существует такое $\lambda$, что $\operatorname{det}(\lambda A+B) \neq 0$; в противном случае $(m \neq n$ или $\operatorname{det}(\lambda A+B) \equiv 0)$ пучок называют сингулярным.

Определение 2 (см. [7]). Будем говорить, что пучок $(n \times n)$-матриц $\lambda A(t)+B(t)$, где $\lambda-$ скалярный параметр, удовлетворяет критерию «ранг-степенъ» (имеет индекс 1 ) на отрезке $[0,1]$, если

$$
\begin{gathered}
\operatorname{rank} A(t)=k=] \text { const } \quad \forall t \in[0,1] ; \\
\operatorname{det}(\lambda A(t)+B(t))=a_{0}(t) \lambda^{k}+a_{1}(t) \lambda^{k-1}+\cdots+a_{k}(t), \quad a_{0}(t) \neq 0 \quad \forall t \in[0,1] .
\end{gathered}
$$

Лемма 1 (см. [2]). Если пучок матрии, $\lambda A(t)+B(t)$ удовлетворяет критерию «ранг-степенъ» на отрезке $[0,1]$, то матрица $A(t)+\left(E-A^{+}(t) A(t)\right) B(t)$ является невырожденной для всех $t \in[0,1]$.

Здесь и всюду в дальнейшем $E$ - единичная матрица, $A^{+}(t)$ - псевдообратная матрица (матрица Мура-Пенроуза) к $A(t)$. Приведем одно из свойств $A^{+}(t)$ (см., например, [1]):

$$
V(t) A(t) \equiv 0,
$$

где $V(t)=E-A(t) A^{+}(t)$.

Если элементы матрицы $A(t)$ - непрерывные функции, то элементы $A^{+}(t)$ будут непрерывными тогда и только тогда, когда матрица $A(t)$ имеет постоянный ранг на заданном отрезке.

Определение 3 (см. [3,5]). Матричный полином $(n \times n)$-матриц $\lambda A(t)+\mu B(t)+C(t)$, где $\lambda$, $\mu$-скалярные параметры, имеет простую структуру на отрезке $[0,1]$, если

$$
\begin{gathered}
\operatorname{rank} A(t)=k=\mathrm{const} \quad \forall t \in[0,1] \\
\operatorname{rank}(A(t) \mid B(t))=k+l=\mathrm{const} \quad \forall t \in[0,1] ; \\
\operatorname{det}(\lambda A(t)+\mu B(t)+C(t))=a_{0}(t) \lambda^{k} \mu^{l}+\ldots, \quad a_{0}(t) \neq 0 \quad \forall t \in[0,1] .
\end{gathered}
$$


Лемма 2 (см. [3]). Если матричный полином $\lambda A(t)+\mu B(t)+C(t)$ имеет простую структуру на отрезке $[0,1]$ и элементы матрии, принадлежат классу $C_{[0,1]}^{m}$, то существуют такие невырожденные $(n \times n)$-матрицы $R(t), S(t), A(t), B(t)$ с элементами из $C_{[0,1]}^{m}$, что

$$
\begin{aligned}
& R(t)(\lambda A(t)+\mu B(t)+C(t)) S(t)= \\
&=\lambda\left(\begin{array}{ccc}
E_{k} & 0 & 0 \\
0 & 0 & 0 \\
0 & 0 & 0
\end{array}\right)+\mu\left(\begin{array}{ccc}
J_{1}(t) & 0 & J_{2}(t) \\
0 & E_{l} & 0 \\
0 & 0 & 0
\end{array}\right)+\left(\begin{array}{ccc}
C_{1}(t) & C_{2}(t) & 0 \\
C_{3}(t) & C_{4}(t) & 0 \\
0 & 0 & E_{n-k-l}
\end{array}\right),
\end{aligned}
$$

где $J_{1}(t), J_{2}(t), C_{i}(t), i=1,2,3,4$ и 0 -блочные и нулевые матрицы подходящих размеров.

Лемма 3 (см. [4]). Если матричный полином $\lambda A(t)+\mu B(t)+C(t)$ имеет простую структуру на отрезке $[0,1]$, элементы матрии, принадлежат классу $C_{[0,1]}^{m}$, то $R(t) A(t), R(t) B(t), R(t) C(t)$ именот блочный вид

$$
R(t) A(t)=\left(\begin{array}{c}
A_{1}(t) \\
0 \\
0
\end{array}\right), \quad R(t) B(t)=\left(\begin{array}{c}
B_{1}(t) \\
B_{2}(t) \\
0
\end{array}\right), \quad R(t) C(t)=\left(\begin{array}{c}
C_{1}(t) \\
C_{2}(t) \\
C_{3}(t)
\end{array}\right),
$$

где $A_{1}(t), B_{1}(t), C_{1}(t)-(k \times n)$-матричьь, $B_{2}(t), C_{2}(t)-(l \times n)$-матрицы, $C_{3}(t)-((n-k-l) \times n)$ матрица и

$$
\operatorname{det}\left(\begin{array}{l}
A_{1}(t) \\
B_{2}(t) \\
C_{3}(t)
\end{array}\right) \neq 0 \quad \forall t \in[0,1]
$$

Отметим, что если матричный пучок $\lambda A(t)+B(t)$ удовлетворяет критерию «ранг-степень», то матричный полином $\lambda A(t)+\mu B(t)+C(t)$ будет иметь простую структуру при любой $(n \times n)$ матрице $C(t)$.

Приведем результат, относящийся к ДАУ первого порядка. Рассмотрим задачу с корректно заданным начальным условием

$$
B(t) x^{\prime}(t)+F(x(t), t)=0, \quad t \in[0,1], \quad x(0)=a,
$$

где $B(t)$ - тождественно вырожденная $(n \times n)$-матрица, $F(\cdot)$ - $n$-мерная вектор-функция.

Характеристикой сложности задачи (5) является понятие индекса, которое имеет несколько определений (см. $[7,9,10])$. Приведем одно из них.

Определение 4 (см. [6]). Будем говорить, что задача (5) имеет индекс дифферениирования $r$, если $r$ - минимальное число аналитических преобразований и дифференцирований, необходимых для того, чтобы из (5) и первых $r$ ее производных выделить в окрестности начальной точки классическую систему ОДУ вида $x^{\prime}(t)+\mathcal{F}(x(t), t)=0$.

Индекс $r$ показывает, что решение данной системы ДАУ зависит от первых $r-1$ производных входных данных. Системам ОДУ первого порядка, разрешенным относительно производной, приписывают нулевой индекс.

Как правило, для исследования ДАУ высокого порядка

$$
A(t) x^{(p)}(t)+F_{1}\left(x^{(p-1)}(t), t\right)+F_{2}\left(x^{(p-2)}(t), t\right)+\cdots+F_{p}(x(t), t)=0, \quad t \in[0,1],
$$

где $A(t)$ - тождественно вырожденная $(n \times n)$-матрица, $F_{j}(\cdot)-n$-мерные вектор-функции, применяют следующий прием: вводят новую переменную

$$
y(t)=\left(x^{(p-1)}(t)^{T}, x^{(p-2)}(t)^{T}, \ldots, x^{(p-1)}(t)^{T}\right)^{T}
$$

и переписывают данную задачу в виде ДАУ первого порядка. Для полученной таким образом системы применяют технику, разработанную для ДАУ первого порядка. В этом случае начальные условия для ДАУ высокого порядка переходят в начальные условия для полученной системы первого порядка. Такой подход обладает двумя недостатками:

(а) увеличивает размерность полученной системы ДАУ; 
(b) индекс ДАУ первого порядка увеличивается и, как правило, не дает информации о сложности исходной системы ДАУ высокого порядка.

Проиллюстрируем это на примерах.

Пример 1. Простейшее уравнение

$$
b(t) x(t)=f(t), \quad b(t) \neq 0 \quad \forall t \in[0,1],
$$

где $x(t)$ - искомая, а $b(t), f(t)$ - заданные функции, перепишем в виде

$$
0 \times x^{(p)}(t)+b(t) x(t)=f(t)
$$

где $p$ - целое неотрицательное число. С учетом обозначения $y(t)=\left(x^{(p-1)}(t), x^{(p-2)}(t), \ldots, x(t)\right)^{T}$ последнее уравнение примет векторно-матричный вид

$$
\mathcal{A} y^{\prime}(t)+\mathcal{B}(t) y(t)=\psi(t), \quad t \in[0,1]
$$

где $((p+1) \times(p+1))$-матрицы $\mathcal{A}(t)$ и $\mathcal{B}(t)$ определены по правилу

$$
\mathcal{A}=\left(\begin{array}{ccccccccc}
0 & 1 & 0 & 0 & \ldots & \ldots & \ldots & 0 & 0 \\
0 & 0 & 1 & 0 & \ldots & \ldots & \ldots & 0 & 0 \\
\ldots & \ldots & \ldots & \ldots & \ldots & \ldots & \ldots & 1 & 0 \\
0 & 0 & 0 & 0 & \ldots & \ldots & \ldots & 0 & 0
\end{array}\right), \quad \begin{aligned}
& \mathcal{B}(t)=\operatorname{diag}(-1,-1, \ldots,-1, b(t)), \\
& \psi(t)=(0,0, \ldots, 0, f(t))^{T} .
\end{aligned}
$$

Размерность полученной ДАУ первого порядка увеличилась в $p+1$ раз, индекс этой системы равен $p$.

Решение данной задачи находим по известной формуле (см. [1])

$$
y(t)=\sum_{j=0}^{p} T^{j} \Psi(t),
$$

где действие оператора $T$ на вектор-функцию $\Psi(t)$ определено по правилу

$$
T \Psi(t)=\mathcal{A} \frac{d}{d t}, \quad T^{0} \Psi(t)=\Psi(t), \quad \Psi(t)=\left(0,0, \ldots, 0, \frac{f(t)}{b(t)}\right)^{T} .
$$

Пример 2. Аналогичные рассуждения можно провести и для весьма простой, как с точки зрения качественной теории, так и с точки зрения вычислительной математики, системы ДАУ

$$
\left(\begin{array}{ll}
1 & t \\
0 & 0
\end{array}\right)\left(\begin{array}{l}
x_{1}(t) \\
x_{2}(t)
\end{array}\right)^{(p)}+\left(\begin{array}{cc}
b_{1}(t) & b_{2}(t) \\
0 & 1
\end{array}\right)\left(\begin{array}{l}
x_{1}(t) \\
x_{2}(t)
\end{array}\right)=\left(\begin{array}{l}
f_{1}(t) \\
f_{2}(t)
\end{array}\right), \quad t \in[0,1]
$$

Точно так же, как в примере ??, введя обозначение

$$
y(t)=\left(x^{(p-1)}(t), x^{(p-2)}(t), \ldots, x(t)\right)^{T}, \quad x(t)=\left(x_{1}(t), x_{2}(t)\right)^{T}
$$

и переписав систему в виде ДАУ первого порядка, видим, что полученная система имеет размерность $2 p$, а ее индекс равен $p$.

Отметим, что регулярность (сингулярность) матричных пучков $\lambda A(t)+B(t), \lambda A(t)+\partial F(x, t) / \partial x$ (в окрестности начальной точки $(a, 0))$ не дает информации о единственности решения исходной задачи. Известно (см. [1]), что для линейных ДАУ первого порядка с постоянными матричными коэффициентами единственность решения начальной задачи гарантирует регулярность матричного пучка. Для ДАУ порядка 2 и выше это не так. Для иллюстрации приведем пример.

Пример 3. Рассмотрим ДАУ

$$
\begin{gathered}
\left(\begin{array}{ll}
1 & 0 \\
0 & 0
\end{array}\right)\left(\begin{array}{l}
x_{1}^{\prime \prime}(t) \\
x_{2}^{\prime \prime}(t)
\end{array}\right)+\left(\begin{array}{ll}
0 & 1 \\
1 & 0
\end{array}\right)\left(\begin{array}{l}
x_{1}^{\prime}(t) \\
x_{2}^{\prime}(t)
\end{array}\right)+\left(\begin{array}{ll}
0 & 0 \\
0 & 1
\end{array}\right)\left(\begin{array}{l}
x_{1}(t) \\
x_{2}(t)
\end{array}\right)=\left(\begin{array}{l}
0 \\
0
\end{array}\right), \quad t \in[0,1], \\
x(0)=x^{\prime}(0)=0, \quad x_{1}^{\prime}(0)=-x_{2}(0)=x_{2}^{\prime}(0)=0 .
\end{gathered}
$$

Легко убедиться, что решением данной задачи является вектор-функция $\left(-\rho(t), \rho^{\prime}(t)\right)^{T}$, где $\rho(t)-$ произвольная достаточно гладкая функция, удовлетворяющая условиям $\rho(0)=\rho^{\prime}(0)=\rho^{\prime \prime}(0)=0$. $\mathrm{B}$ данном примере матричные пучки $\lambda A(t)+B(t), \lambda A(t)+\partial F(x, t) / \partial x$ являются регулярными. 
Пример 4. Рассмотрим ДАУ

$$
\left(\begin{array}{cc}
1 & a(t) \\
0 & 0
\end{array}\right)\left(\begin{array}{c}
x_{1}^{\prime \prime}(t) \\
x_{2}^{\prime \prime}(t)
\end{array}\right)+\left(\begin{array}{cc}
0 & \alpha \\
1 & a(t)
\end{array}\right)\left(\begin{array}{l}
x_{1}^{\prime}(t) \\
x_{2}^{\prime}(t)
\end{array}\right)=\left(\begin{array}{l}
f_{1}(t) \\
f_{2}(t)
\end{array}\right), \quad t \in[0,1]
$$

где $a(t), f_{1}(t), f_{2}(t)$ - достаточно гладкие функции, $\alpha$-скалярный параметр. Из второго уравнения находим $x_{1}^{\prime}(t)=f_{2}(t)-a(t) x_{2}^{\prime}(t)$. Подставляя это выражение в первое уравнение, получим

$$
\left(\alpha-a^{\prime}(t)\right) x_{2}^{\prime}(t)=f_{1}(t)-f_{2}^{\prime}(t) .
$$

При $\alpha-a^{\prime}(t)=0$ и $f_{1}(t)-f_{2}^{\prime}(t) \neq 0$ при некоторых значениях $t \in[0,1]$ данная задача не имеет решения.

При $\alpha=0$ пучок матриц будет сингулярным. Вычислим индекс данной системы. Введем обозначение $x(t)=(u(t), v(t))^{T}$. Дифференцируя второе уравнение, получим систему

$$
\left(\begin{array}{cc}
1 & a(t) \\
1 & a(t)
\end{array}\right)\left(\begin{array}{l}
x_{1}^{\prime \prime}(t) \\
x_{2}^{\prime \prime}(t)
\end{array}\right)+\left(\begin{array}{cc}
0 & \alpha \\
0 & a^{\prime}(t)
\end{array}\right)\left(\begin{array}{l}
x_{1}^{\prime}(t) \\
x_{2}^{\prime}(t)
\end{array}\right)=\left(\begin{array}{c}
f_{1}(t) \\
f_{2}^{\prime}(t)
\end{array}\right)
$$

Вычитая из второго уравнения первое, находим

$$
\left(\begin{array}{cc}
1 & a(t) \\
0 & 0
\end{array}\right)\left(\begin{array}{l}
x_{1}^{\prime \prime}(t) \\
x_{2}^{\prime \prime}(t)
\end{array}\right)+\left(\begin{array}{cc}
0 & \alpha \\
0 & a^{\prime}(t)-\alpha
\end{array}\right)\left(\begin{array}{l}
x_{1}^{\prime}(t) \\
x_{2}^{\prime}(t)
\end{array}\right)=\left(\begin{array}{c}
f_{1}(t) \\
f_{2}^{\prime}(t)-f_{1}(t)
\end{array}\right)
$$

Дифференцируя второе уравнение последней системы, получаем

$$
\left(\begin{array}{cc}
1 & a(t) \\
0 & a^{\prime}(t)-\alpha
\end{array}\right)\left(\begin{array}{c}
x_{1}^{\prime \prime}(t) \\
x_{2}^{\prime \prime}(t)
\end{array}\right)+\left(\begin{array}{cc}
0 & \alpha \\
0 & a^{\prime \prime}(t)
\end{array}\right)\left(\begin{array}{c}
x_{1}^{\prime}(t) \\
x_{1}^{\prime}(t)
\end{array}\right)=\left(\begin{array}{c}
f_{1}(t) \\
f_{2}^{\prime \prime}(t)-f_{1}^{\prime}(t)
\end{array}\right)
$$

которая сводится к системе ОДУ, если $a^{\prime}(t)-\alpha \neq 0$ для всех $t \in[0,1]$. Таким образом, после двух дифференцирований и элементарных преобразований мы свели задачу к системе ОДУ, т.е. индекс системы равен двум.

Приведем вид решения данной задачи. Из второго уравнения находим $u(t)=g(t)-a(t) v(t)$. Подставляя это выражение в первое уравнение, получим

$$
u(t)=\frac{q(t)-g^{\prime}(t)}{\alpha-a^{\prime}(t)} .
$$

Если $a^{\prime}(t) \neq \alpha$ для всех $t \in[0,1]$, то задача имеет единственное решение, зависящее от $a(t), q(t)$, $g(t), a^{\prime}(t)$ и $g^{\prime}(t) ;$ при этом решение не зависит от начальных условий $(2)$.

Итак, последние два примера иллюстрируют тот факт, что для исследования ДАУ порядка 2 и выше привлечение теории матричных пучков не всегда возможно.

В следующем разделе мы выделим задачи (1)-(2), для которых будут сформулированы условия, гарантирующие существование и единственность решения.

3. Условия существования единственного решения. Приведем достаточные условия существования единственного достаточно гладкого решения рассматриваемых задач.

Теорема 1. Пусть для задачи (1)-(2) выполнены следующие условия:

(1) элементы входных данных достаточно гладкие;

(2) начальные данные заданы корректно;

(3) пучок матрии, $\lambda A(t)+B(t)$ удовлетворяет критерию «ранг-степенъ».

Тогда в окрестности начальной точки решение задачи существует и единственно.

Доказательство. Доказательство теоремы основано на следующем наблюдении: подействовав на задачу оператором

$$
E+\left(E-A(t) A^{+}(t)\right) \frac{d}{d t},
$$

в силу леммы 1 получим систему ОДУ второго порядка с невырожденной (при всех $t \in[0,1]$ ) матрицей перед второй производной. В силу первого условия теоремы полученная задача имеет единственное решение из класса $C_{[0,1]}^{2}$ в окрестности начальной точки. 
Теорема 2. Пусть для задачи (1)-(2) выполнены следующие условия:

(1) элементы входных данных достаточно гладкие;

(2) начальные данные заданы корректно;

(3) $B(t)-$ тождественно нулевая матрича;

(4) пучок матрии, $\lambda A(t)+\partial F(x, t) / \partial x$ удовлетворяет критерию «ранг-степень» в начальной точке.

Тогда в окрестности начальной точки решение задачи существует и единственно.

Доказательство. Действуя на данную задачу оператором

$$
E+\left(E-A(t) A^{+}(t)\right)\left(\frac{d}{d t}\right)^{2},
$$

в силу леммы 1 получим систему ОДУ второго порядка с невырожденной матрицей перед второй производной в окрестности начальной точки. В силу первого условия теоремы полученная задача имеет единственное решение из класса $C_{[0,1]}^{2}$ в окрестности начальной точки.

Рассмотрим частный случай задачи (1)-(2):

$$
A x^{\prime \prime}(t)+B x^{\prime}(t)+C x(t)=f(t), \quad t \in[0,1], \quad x(0)=a, \quad x^{\prime}(0)=b,
$$

где $A, B, C-(n \times n)$-матрицы с постоянными коэффициентами. При исследовании таких задач на предмет единственности решения важную роль играет матричный полином вида $\lambda^{2} A+\lambda B+C$.

Введем обозначение $y(t)=\left(x^{\prime}(t)^{T}, x(t)^{T}\right)^{T}$ и перепишем задачу (10) в виде ДАУ первого порядка:

где $\mathcal{A}, \mathcal{B}-(2 n \times 2 n)$-блочные матрицы вида

$$
\mathcal{A} y(t)+\mathcal{B} y(t)=\xi(t), \quad t \in[0,1], \quad y(0)=\left(b^{T}, a^{T}\right)^{T},
$$

$$
\mathcal{A}=\left(\begin{array}{cc}
A & B \\
0 & E
\end{array}\right), \quad \mathcal{B}=\left(\begin{array}{cc}
0 & C \\
-E & 0
\end{array}\right)
$$

Известно (см. [1]), что если задача (11) имеет решение (т.е. начальные данные заданы корректно), то регулярность матричного пучка $\lambda \mathcal{A}+\mathcal{B}$ гарантирует его единственность.

Матричный пучок $\lambda \mathcal{A}+\mathcal{B}$ имеет блочный вид

$$
\lambda \mathcal{A}+\mathcal{B}=\lambda\left(\begin{array}{cc}
\lambda A & \lambda B+C \\
-E & \lambda E
\end{array}\right) .
$$

Используя блочный алгоритм Гаусса для вычисления определителя $\lambda \mathcal{A}+\mathcal{B}$ (см. [5]), получим

$$
\operatorname{det}(\lambda \mathcal{A}+\mathcal{B})=\operatorname{det}\left(\begin{array}{cc}
0 & \lambda^{2} A+\lambda B+C \\
-E & \lambda E
\end{array}\right)=-\operatorname{det}\left(\lambda^{2} A+\lambda B+C\right) .
$$

Таким образом, задача (10) будет иметь единственное решение тогда и только тогода, когда матричный полином $\lambda^{2} A+\lambda B+C$ регулярен, т.е. существует такое $\lambda$, что $\operatorname{det}\left(\lambda^{2} A+\lambda B+C\right) \neq 0$.

Сформулируем теперь достаточные условия существования единственного решения задачи (1)(2). Введем обозначение $C(x, t)=\partial F(x, t) / \partial x$.

Теорема 3. Пусть для задачи (1)-(2) выполнены следуюшие условия:

(1) элементы входных данных дважды непреравно дифберениируемы;

(2) $\operatorname{rank} A(0)=\operatorname{rank}\left(A(0) \mid B(0) x^{\prime}(0)+F\left(x_{0}, 0\right)\right)$;

(3) матричный полином $\lambda A(t)+\nu B(t)+C(x, t)$ имеет простую структуру в окрестности начальной точки.

Тогда в окрестности начальной точки определено единственное решение задачи (1)-(2).

Доказательство. Подставляя в (1) начальные значения $x(0)=a,\left.x^{\prime}(t)\right|_{t=0}=b$, получим систему линейных алгебраических уравнений

$$
A(0) x^{\prime \prime}(0)+B(0) x^{\prime}(0)+F\left(x_{0}, 0\right)=0 .
$$

Разрешимость этой системы гарантируется вторым условием теоремы. 
В силу леммы 3 существует такая матрица $R(t)$ с дважды непрерывно дифференцируемыми элементами, что при умножении исходной системы на $R(t)$ получаются системы уравнений

$$
\begin{gathered}
A_{1}(t) x^{\prime \prime}(t)+B_{1}(t) x^{\prime}(t)+F_{1}(x(t), t)=0, \\
B_{2}(t) x^{\prime}(t)+F_{2}(x(t), t)=0, \\
F_{3}(x(t), t)=0,
\end{gathered}
$$

где $A_{1}(t), B_{1}(t)-(k \times n)$-матрицы, $B_{2}(t)-(l \times n)$-матрица, вектор-функции $F_{1}(\cdot), F_{2}(\cdot), F_{3}(\cdot)$ имеют размерности $k, l$ и $n-k-l$ соответственно, $R(t)$ - матрица из леммы 3.

Дифференцируя (15) один раз по $t$, а (16) - два раза по $t$, в силу первого условия теоремы, получим систему ОДУ второго порядка с матрицей перед производной вида

$$
\left(\begin{array}{c}
A_{1}(t) \\
B_{2}(t) \\
C_{3}(x(t), t)
\end{array}\right)
$$

где $C_{3}(\cdot)=\partial F_{3}(x, t) / \partial x$.

В силу третьего условия теоремы и леммы 3, данная матрица является невырожденной в начальной точке. Так как функция определителя является непрерывной, то и в некоторой окрестности этой точки данная матрица будет невырожденной, т.е. рассматриваемая система приводима к стандартной задаче Коши для ОДУ второго порядка, которая, в силу первого условия теоремы, будет иметь единственное решение.

4. Примеры. Проиллюстрируем приведенные выше теоремы несколькими примерами.

Пример 5. Рассмотрим задачу

$$
\begin{gathered}
\left(\begin{array}{cc}
-t^{2} / 2 & 0 \\
0 & 0
\end{array}\right)\left(\begin{array}{l}
x_{1}^{\prime \prime}(t) \\
x_{2}^{\prime \prime}(t)
\end{array}\right)+\left(\begin{array}{cc}
0 & 0 \\
0 & 0,5-t
\end{array}\right)\left(\begin{array}{l}
x_{1}^{\prime}(t) \\
x_{2}^{\prime}(t)
\end{array}\right)+\left(\begin{array}{l}
x_{1}(t) \\
x_{2}(t)
\end{array}\right)=\left(\begin{array}{l}
x_{1}(t) \\
x_{2}(t)
\end{array}\right), \\
x_{1}(0)=x_{2}(0)=x_{1}^{\prime}(0)=x_{2}^{\prime}(0)=0, t \in[0,1] .
\end{gathered}
$$

Легко убедиться, что помимо тривиального решения данная задача имеет множество решений вида

$$
x_{1}(t)=c_{1} t^{2}, \quad x_{2}(t)= \begin{cases}0, & t \in[0 ; 0,5], \\ c_{2}(t-0,5), & t \in[0,5 ; 1] .\end{cases}
$$

$\mathrm{B}$ точках $t=0, t=0,5$ происходит нарушение условий теоремы $1:$ при $t=0$ ранг матрицы $A(t)$ равен нулю (при остальных значениях единице), а при $t=0,5$ нарушается условие равенства ранга матрицы $A(t)$ степени полинома $\operatorname{det}(\lambda A(t)+B(t))$, т.е. в этой точке $\operatorname{det}(\lambda A(t)+B(t))=$ $\lambda t(0,5-t)=0$ при $t=0,5$.

Таким образом, через точки, в которых нарушаются условия теоремы 1 , может проходить несколько решений либо решение будет иметь разрывы.

Пример 6. Рассмотрим уравнения

$$
\begin{gathered}
a_{11}(t) x_{1}^{\prime \prime}(t)+b_{11}(t) x_{1}^{\prime}+b_{13}(t) x_{3}^{\prime}(t)+c_{11}(t) x_{1}(t)=f_{1}(t), \\
b_{22}(t) x_{2}^{\prime}(t)+c_{22}(t) x(t)=f_{2}(t), \quad x_{3}^{2}=-t .
\end{gathered}
$$

где $a_{11}(t), b_{11}(t), b_{13}(t), c_{11}(t), f_{1}(t), b_{22}(t), c_{22}(t), f_{2}(t)$ - заданные непрерывные функции. Точки, в которых функции $a_{11}(t), b_{22}(t)$ обращаются в нуль, будут сингулярными точками для ОДУ второго и первого порядка соответственно. В этих точках решение может быть разрывным либо через них проходит множество решений. Третье уравнение не имеет решения в классе вещественных функций. 
Проведем исследование данного примера, используя результаты теоремы 3. Перепишем эти уравнения в виде ДАУ (1):

$$
\left(\begin{array}{ccc}
a_{11}(t) & 0 & 0 \\
0 & 0 & 0 \\
0 & 0 & 0
\end{array}\right)\left(\begin{array}{l}
x_{1}^{\prime \prime}(t) \\
x_{2}^{\prime \prime}(t) \\
x_{3}^{\prime \prime}(t)
\end{array}\right)+\left(\begin{array}{ccc}
b_{11}(t) & 0 & b_{13}(t) \\
0 & b_{22}(t) & 0 \\
0 & 0 & 0
\end{array}\right)\left(\begin{array}{l}
x_{1}^{\prime}(t) \\
x_{2}^{\prime}(t) \\
x_{3}^{\prime}(t)
\end{array}\right)+\left(\begin{array}{c}
c_{11}(t) x_{1}(t)-f_{1}(t) \\
c_{22}(t) x_{2}(t)-f_{2}(t) \\
x_{3}^{2}(t)+t
\end{array}\right)=\left(\begin{array}{l}
0 \\
0 \\
0
\end{array}\right) .
$$

Точки, в которых функция $a_{11}(t)$ обращается в нуль, будут точками перемены ранга матрицы $A(t)$, т.е. в этих точках происходит нарушение первого условия теоремы. Точки, в которых функция $b_{22}(t)$ обращается в нуль, будут точками перемены ранга матрицы $(A(t) \mid B(t)$, т.е. в этих точках происходит нарушение второго условия теоремы. Наконец в точке $t=0$ происходит нарушение третьего условия теоремы: в этой точке

$$
\operatorname{det}(\lambda A(t)+\mu B(t)+C(x(t), t))=2 x_{3}\left(\lambda a_{11}(t)+\mu b_{11}(t)+c_{11}(t)\right)\left(\mu b_{22}(t) c_{22}(t)\right)=0 .
$$

Отметим, что теорема 3 дает только достаточные условия существования решения исходной задачи. Эти условия далеки от необходимых. Приведем весьма простой пример:

$$
\left(\begin{array}{ccc}
0 & a_{12}(t) & 0 \\
0 & 0 & a_{23}(t) \\
0 & 0 & 0
\end{array}\right)\left(\begin{array}{l}
x_{1}^{\prime \prime}(t) \\
x_{2}^{\prime \prime}(t) \\
x_{3}^{\prime \prime}(t)
\end{array}\right)+\left(\begin{array}{ccc}
0 & b_{12}(t) & 0 \\
0 & 0 & b_{23}(t) \\
0 & 0 & 0
\end{array}\right)\left(\begin{array}{l}
x_{1}^{\prime}(t) \\
x_{2}^{\prime}(t) \\
x_{3}^{\prime}(t)
\end{array}\right)+\left(\begin{array}{c}
x_{1}(t) \\
x_{2}(t) \\
x_{3}(t)-f(t)
\end{array}\right)=\left(\begin{array}{l}
0 \\
0 \\
0
\end{array}\right) .
$$

В этом случае решение (при достаточно гладких входных данных) системы не зависит от начальных данных и находится по формуле

$$
\begin{aligned}
& x_{1}(t)=a_{12}(t)\left(b_{23}(t) f^{\prime}(t)+a_{23}(t) f^{\prime \prime}(t)\right)^{\prime \prime}+b_{12}(t)\left(b_{23}(t) f^{\prime}(t)+a_{23}(t) f^{\prime \prime}(t)\right)^{\prime}, \\
& x_{2}(t)=-b_{23}(t) f^{\prime}(t)-a_{23}(t) f^{\prime \prime}(t), \\
& x_{3}(t)=f(t) .
\end{aligned}
$$

Легко проверить, что для данного примера условия теоремы не выполнены.

5. Заключение. В настоящей работе приведены достаточные условия существования единственного достаточно гладкого решения начальной задачи для квазилинейных ДАУ второго порядка. Обоснование приведено в терминах матричных полиномов. Не представляет принципиальных затруднений обобщить данный результат для ДАУ более высокого порядка (начальная задача), в том числе и для нелинейных систем. Сформулированные условия можно проверять на компьютере, используя современные программы нахождения рангов матриц и символьного вычисления определителя.

В дальнейшем планируется разработка численных методов решения рассмотренных задач с учетом специфики матричных полиномов.

\section{СПИСОК ЛИТЕРАТУРЫ}

1. Бояринцев Ю. Е. Регулярные и сингулярные системы линейных обыкновенных дифференциальных уравнений. - Новосибирск: Наука, 1980.

2. Булатов М. В. О преобразовании алгебро-дифференциальных систем уравнений// Ж. вычисл. мат. мат. физ. - 1994. - 34, № 3. - С. 360-372.

3. Булатов М. В., Минг-Гонг Ли Применение матричных полиномов к исследованию линейных дифференциально-алгебраических уравнений высокого порядка// Диффер. уравн. - 2008. — 44, № 10. - C. $1299-1306$.

4. Булатов М. В., Чистлкова Е. В. Об одном семействе вырожденных интегродифференциальных уравнений// Ж. вычисл. мат. мат. физ. - 2011. - 51, № 9. - С. 1665-1673.

5. Гантмахер Ф. Р. Теория матриц. - М.: Наука, 1986.

6. Хайрер Э., Ваннер $Г$. Решение обыкновенных дифференциальных уравнений. Жесткие и дифференциально-алгебраические задачи. - М.: Мир, 1999.

7. Чистлков В. Ф. Алгебро-дифференциальные операторы с конечномерным ядром. - Новосибирск: Наука, 1996. 
8. Bock H. G., Schloder J. P., Schulz V. H. Differential-Algebraic Equations and Their Connections to Optimization. - Heidelberg, 1996.

9. Brenan K. F., Campbell S. L., Petzold L. R. Numerical Solution of Initial-Value Problems in DifferentialAlgebraic Equations. - Philadelphia: SIAM, 1996.

10. Lamour R., März R., Tischendorf C. Differential-Algebraic Equations: A Projector Based Analysis. Berlin-Heidelberg: Springer-Verlag, 2013.

Булатов Михаил Валерьянович

Институт динамики систем и теории управления им. В. М. Матросова

Сибирского отделения РАН, Иркутск;

Бурятский государственный университет

E-mail: mvbul@icc.ru

Соловарова Любовь Степановна

Институт динамики систем и теории управления им. В. М. Матросова

Сибирского отделения РАН, Иркутск

E-mail: soleilu@mail.ru 